\title{
Evaluation of a novel fluorescent nanobeacon for targeted imaging of Thomsen-Friedenreich associated colorectal cancer
}

This article was published in the following Dove Press journal:

International Journal of Nanomedicine

2 March 2017

Number of times this article has been viewed

Hiroshi Nakase, ${ }^{1,2}$ Shinji Sakuma, ${ }^{3}$ Takumi Fukuchi, ${ }^{4}$ Takuya Yoshino, ${ }^{1,5}$ Kohta Mohri, ${ }^{3}$ Kohei Miyata, ${ }^{3,6}$ Hironori Kumagai, ${ }^{6}$ Ken-Ichiro Hiwatari, ${ }^{6}$ Kazufumi Tsubaki, ${ }^{6}$ Tetsuya Ikejima, ${ }^{6}$ Etsuo Tobita, ${ }^{6}$ Meiying Zhu, ${ }^{7}$ Kevin J Wilson, ${ }^{7}$ Kay Washington, ${ }^{8}$ John C Gore, ${ }^{7,9-13}$ Wellington Pham ${ }^{7,9-12,14}$

'Division of Endoscopy, Kyoto University Hospital, Kyoto, Japan; ${ }^{2}$ Department of Gastroenterology and Hepatology, Sapporo Medical University School of Medicine, Sapporo, Hokkaido, Japan; ${ }^{3}$ Faculty of Pharmaceutical Sciences, Setsunan University, Hirakata, Osaka, Japan; ${ }^{4}$ Gastroenterology and Hepatology, Osakafu Saiseikai Nakatsu Hospital, Osaka, Japan; ${ }^{5}$ Division of

Inflammatory Bowel Disease, Digestive Disease Center, Kitano Hospital, Osaka, Japan; ${ }^{6}$ Advanced Materials R\&D Laboratory, ADEKA Corp., Tokyo, Japan; ${ }^{7}$ Department of Radiology, Vanderbilt University Institute of Imaging Science, ${ }^{8}$ Division of Gastrointestinal and Hepatic Pathology, Vanderbilt School of Medicine, ${ }^{9}$ Department of Radiology and Radiological Sciences, ${ }^{10}$ Department of Biomedical Engineering, "Vanderbilt Ingram Cancer Center, Vanderbilt School of Medicine, ${ }^{12}$ Vanderbilt Brain Institute, ${ }^{13}$ Molecular Physiology and Biophysics, ${ }^{14}$ Vanderbilt Institute of Chemical Biology, Vanderbilt University, Nashville, TN, USA

Correspondence: Wellington Pham Vanderbilt University Institute of Imaging Science, II6I, 2 Ist Avenue South, AAA 3117 , Nashville, TN 37232, USA

Tel + I 6I5936762|

Fax + I 6153220734

Email wellington.pham@vanderbilt.edu Shinji Sakuma

Faculty of Pharmaceutical Sciences, Setsunan University, 45-I Nagaotoge-cho, Hirakata,

Osaka 573-0I0I, Japan

Tel $+8 \mid 728663$ I 24

Fax +8I 728663126

Email sakuma@pharm.setsunan.ac.jp

Hiroshi Nakase

Department of Gastroenterology and

Hepatology, Sapporo Medical University School of Medicine, S-I, W-16, Chuo-ku, Sapporo,

Hokkaido 060-8543, Japan

Tel $+8 \mid$ I I 6II 21||

Fax +8| || 6|3 |24|

Email hiro_nakase@sapmed.ac.jp
Abstract: The Thomsen-Friedenreich (TF) antigen represents a prognostic biomarker of colorectal carcinoma. Here, using a nanobeacon, the surface of which was fabricated with peanut agglutinin as TF-binding molecules, we demonstrate that the nanobeacon is able to detect TF antigen in frozen and freshly biopsied polyps using fluorescence microscopy. Our results provide important clues about how to detect aberrant colonic tissues in the most timely fashion. Given the versatile application method for this topical nanobeacon, the protocol used in this work is amenable to clinical colonoscopy. Moreover, the prospects of clinical translation of this technology are evident.

Keywords: TF antigen, fluorescence colonoscopy, nanobeacon, colorectal cancer

\section{Background}

Colorectal cancer (CRC) is not only the third most common cancer among adults in the United States, but it is also the second deadliest. ${ }^{1}$ Since the campaign for CRC screening began, the number of CRC-related deaths has been reduced by approximately $30 \%$. With the availability of the state-of-the-art colonoscopy, the detection of CRC is relatively simple, but small adenomatous polyps typically evade colonoscopic detection. Unfortunately, the miss rate of colonoscopy for small and flat polyps is approximately $24 \% .^{2,3}$ Detection and removal of these adenomatous polyps is paramount to controlling the disease and preventing CRC death, since nearly all cases of CRC begin as benign adenomatous polyps, which slowly develop into carcinomas as they collect sufficient genetic materials. Ironically, those blind spot flat polyps often become aggressive forms of CRC. ${ }^{4}$ Fortunately, as it takes several years for adenomas to develop into carcinomas, a perfect window of opportunity for intervention exists before the disease becomes difficult to control as the risk of cancer increases exponentially over time. ${ }^{5}$ Recently, we developed a nanobeacon capable of illuminating those small polyps by using an integrative technique intended to fill that detection gap. The nanobeacon is comprised of polystyrene nanoparticles that have coumarin 6 dyes encapsulated within the core, the surface of which was fabricated with poly ( $N$-vinylacetamide) (PNVA). We coated the surface of each nanobeacon with peanut agglutinin (PNA), which has a high affinity for the TF antigen, a disaccharide Gal- $\beta(1-3)$ GalNAc. In epithelial cells, TF antigen is carried by MUC-1 on the apical surface, while in tumor cells, MUC-1 is posttranslationally modified, resulting in aberrant O-glycosylation, such as TF antigen, a well-defined antigen with a proven link to CRC. ${ }^{6}$ Its expression is also found to correlate with poor prognosis in $\mathrm{CRC}^{7}$ and with a significantly higher risk of developing metastasis. ${ }^{8}$ In keeping with this observation, developing a fundamental 
understanding of the in vivo expression of the TF antigen would yield strategies to achieve effective means of detecting CRC in its early stage. Data obtained from our work with the nanobeacon suggested that TF antigen is an oncodevelopmental cancer-associated antigen, the expression of which correlates with conventional histopathological parameters related to early cancer and malignancy. ${ }^{9,10}$ We performed the previous work on orthotopic CRC animal models and frozen human tissues, but the inability to test the nanobeacon in an authentic colonic microenvironment complicates its eventual translation. For instance, the presence of mucosal substances within the intestinal mucosa may impede binding and thus attenuate the specificity of the nanobeacon. Thus, further testing of this topically applied nanobeacon with minimal logistics amenable to clinical colonoscopy is not a matter of if, but when. Accordingly, there is a strong rationale behind the need to investigate the specificity of the nanobeacon on unfrozen human tissues.

Here, we address for the first time the binding specificity of the nanobeacon on freshly biopsied human colonic specimens in a clinical setting, wherein the nanobeacon was able to discern normal from pathological tissues. Particularly, TF expression is not only strong in cancer tissues, but it is also prominent in the adenoma that makes the topical nanobeacon approach for early detection of CRC feasible.

\section{Methods}

\section{Materials}

Coumarin 6 and PNA were obtained from Sigma-Aldrich (St Louis, MO, USA), while $N$-vinylacetamide (NVA) monomers were kindly provided by Showa Denko Co. (Tokyo, Japan) as a gift. All other chemicals were obtained from commercial sources at reagent grade and used without further purification. The anti-human TF monoclonal antibody was obtained from Abcam (Cambridge, UK) and LifeSpan BioSciences (Seattle, WA, USA).

\section{Tissue collection and reagents}

Before the study, all patients gave their written informed consent according to the guidelines published by the Institutional Review Board. The work done at Vanderbilt University School of Medicine, Osakafu Saiseikai Nakatsu Hospital, and Setsunan University Faculty of Pharmaceutical Sciences, and was approved by Vanderbilt University Institutional Review Board (141940), Setsunan University Ethical Review Committee for Medical and Health Research Involving Human Subjects, and Institutional Review Board at Osakafu Saiseikai Nakatsu Hospital. The anti-human TF monoclonal antibody was obtained from LifeSpan BioSciences, and immunohistochemical (IHC) staining for colorectal section samples was performed using the avidin-biotin-peroxidase complex (ABC) method available in a commercial kit (Vector Laboratories, Burlingame, CA, USA). Imaging was performed on Zeiss (Vanderbilt) and Olympus IX 71 (Osaka) microscopes.

\section{Nanobeacon}

The synthesis of the nanobeacon was modified trenchantly from the past report ${ }^{9}$ including a change in the ratio of the polymers and solvents to improve specificity, safety, and reproducibility for this clinical work. Briefly, a batch of surface coating polymers of PNVA (poly-NVA) and PMA (poly-MA) were generated in a free radical polymerization of NVA or TBMA (tert-butyl methacrylate) monomers with 2-mercaptoethanol (Figure 1A).
A

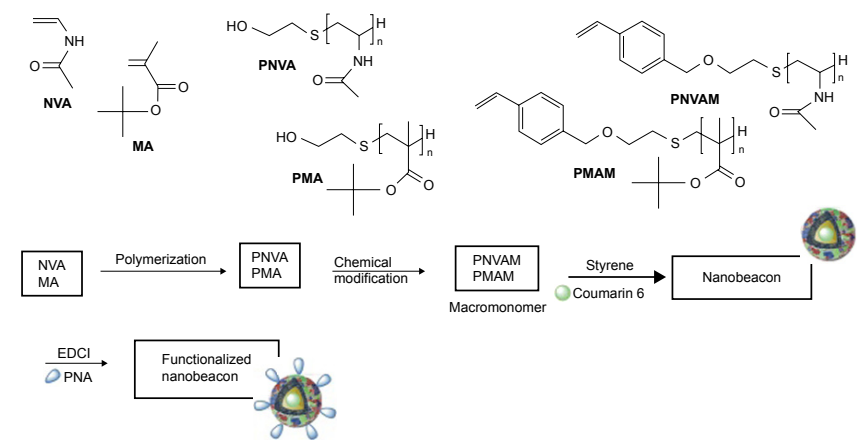

B

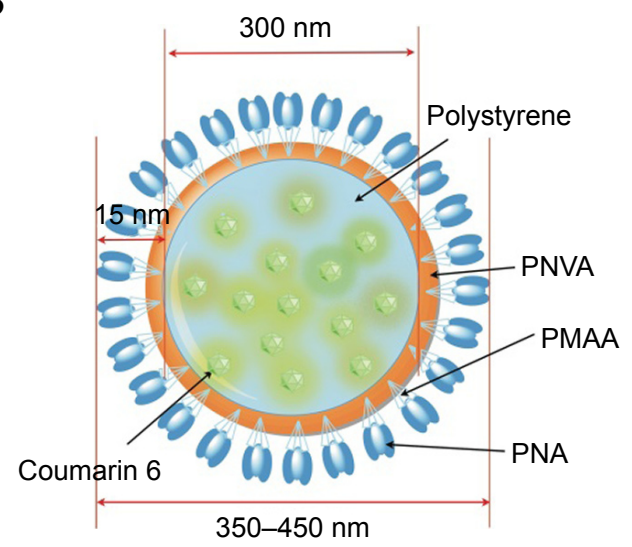

Figure I (A) The overall design of the PNA-laden nanobeacon. The probe is comprised of coumarin 6 dyes encapsulated inside polystyrene polymer core, which was coated with a multicomponent and biocompatible layer to achieve particle integrity and enhance binding specificity. The surface coating polymer consists of PNVA and PMAA polymers on the surface. The former is used to prevent unspecific binding to the colon mucosa, while the latter provides carboxylates for bioconjugation. For the key molecular recognition molecules, the PNA molecules were fabricated on the surface of the nanobeacon by coupling the amino groups on PNA with carboxylates on PMAA; (B) the overall size of the nanobeacon is approximately $350-450 \mathrm{~nm}$. Approximately 200-300 PNA are attached to the nanobeacon through covalent bonding to the PMAA polymer as recognition molecules for TF antigen, while coumarin 6 dye constitutes about $0.05 \%$ of the overall weight of the nanobeacon.

Abbreviations: PMAA, polymethacrylic acid; PNVA, poly(N-vinylacetamide); TF, Thomsen-Friedenreich; PNA, peanut agglutinin. 
The polymers were further modified with $p$-chloromethyl styrene in the presence of tetrabutylphosphonium bromide via the hydroxyl moieties in the polymers' termini to afford the macromonomers, poly(N-vinylacetamide) macromonomer (PNVAM), and poly(methacrylic acid) macromonomer (PMAM). To make the nanobeacon, a PNVAM (1.0 eq in grams), PMAM (1.0 eq), and styrene (2.0 eq) were introduced into a solution of AIBME (2,2'-azobis(2-methylpropionate)) ( $\sim 1.0 \mathrm{~mol} \%$ of the total monomers) and coumarin 6 dye $(0.1 \%$ of the total monomers) in ethanol:water (2:1 ratio). The resulting mixture was stirred for $18 \mathrm{~h}$ at $60^{\circ} \mathrm{C}$. The final product was collected by centrifugation, while the unreacted materials were discarded in the supernatant. The product was further centrifuged several times with an excess amount of water to remove ethanol. Finally, the nanobeacon was dispersed in deuterium-depleted water and lyophilized for future use.

The nanobeacon was functionalized by treating with EDCI (1-ethyl-3-(3-dimethylaminopropyl)carbodiimide) (3 eq) followed by addition of PNA. The reaction was stirred for $24 \mathrm{~h}$ at $4^{\circ} \mathrm{C}$. Then, the PNA-laden nanobeacon dispersion was centrifuged to separate the precipitated product from the unreacted materials three times, and the product was finally dispersed in deuterium-depleted water. Several batches of the PNA-coated nanobeacon were synthesized using the same procedure noted previously and characterized to ensure the reproducibility of our production chemistry (Figure S1). All nanobeacon batches had a concentration of $20 \mathrm{mg} / \mathrm{mL}$ in phosphate buffer saline (PBS) at $\mathrm{pH} 7.2 \pm 0.05$, and all of the physicochemical properties of the nanobeacons were confirmed in each batch. Coating the PNAs on the surface of the nanobeacon increased its size slightly to a thickness of approximately $15 \mathrm{~nm}$ (Figure 1B). In general, all batches consistently had nanobeacons with an overall size of between 350 and $450 \mathrm{~nm}$. Quantitatively, we measured about 200-300 PNA molecules have been fabricated on the surface of the nanobeacon, and this is equivalent to 3-6 $\mu \mathrm{g} / \mathrm{mg}$ nanobeacon, while the core of each nanoparticle contains approximately 0.05 (wt $\%)$ of coumarin 6 dye.

\section{Frozen tissues and imaging process}

Frozen normal and polyp samples ( $n=7$, each) were air dried and fixed with $4 \%$ paraformaldehyde for $10 \mathrm{~min}$ before being exposed to a solution of citric acid buffer ( $\mathrm{pH}$ 6.0). Then, the slides were washed with Dulbecco's phosphate buffer saline (DPBS) before exposure to the nanobeacon $(20 \mathrm{mg} / \mathrm{mL})$ for fluorescence microscopy (Nuance FX, CRI). An FITC channel was used to detect the nanobeacon. Images observed under fluorescence/white visible light were captured with a charge-coupled device (CCD) camera operated via Nuance software (Nuance, Burlington, MA, USA).

\section{Biopsied tissues and imaging process}

Pathological tissues were removed from patients during colonoscopy at either Vanderbilt Medical Center or Osakakafu Saiseikai Nakatsu Hospital, a small portion, approximately $0.3-1.6 \mathrm{~g}$, of which was used for imaging work. In addition, a similar weight of normal colonic tissues was obtained from the same patient as a comparative control counterpart. We used two preparative protocols to stain the tissues before imaging. In the first protocol, freshly removed tissues were briefly washed in PBS solution (1 min) before being dissected into two or more pieces corresponding to testing with different batches of nanobeacons, before being exposed to bovine serum albumin (BSA), as a blocking solution, for $1 \mathrm{~min}$. The tissues were then soaked for $2 \mathrm{~min}$ at room temperature in nanobeacon solutions that ranged in concentration from 2 to $10 \mathrm{mg} / \mathrm{mL}$. To remove any excess nanobeacon solution, the tissues were washed with PBS (1 min) before being exposed under a fluorescent microscope. The process used in the second protocol was identical to that in the first, except that BSA was eliminated in the protocol and with extra washing time in PBS $(4 \times 30 \mathrm{sec}$ each) after tissue exposure to the nanobeacon. All fluorescent microscopy was performed using exposure times that ranged between 1/60 and 1/100 sec. Excitation wavelength was in the range of $470-495 \mathrm{~nm}$, and the collected emission wavelength was $510-550 \mathrm{~nm}$.

\section{Quantitative analysis of fluorescence}

After imaging TF expression in the freshly isolated human colonic tissues using fluorescent microscopy, histogram analysis of the TF expression images was conducted using an algorithm developed within MATLAB (MathWorks, Inc., Natick, MA, USA). To delineate no signal, pixels with intensity below $10 \%$ of the maximum signal were excluded from the computation. Histograms representing the fluorescence signal were created for each image by partitioning the pixel intensity values into 20 equally spaced bins. For each image, a best-fit distribution was computed and overlaid onto the histogram.

\section{Immunohistochemistry}

After fluorescent imaging using a microscope, human tissues were snap frozen in OCT (Tissue-Tek, Fisher Scientific, Hampton, NH, USA) and stored for future use at $-80^{\circ} \mathrm{C}$. The tissues were sectioned into 8-micron slides using a cryostat (Tissue-Tek Cryo3, Sakura Finetek, Alphen aan den Rijn, the Netherlands) and stained with a 1:100 dilution of anti-TF antibodies (LifeSpan BioScience) overnight at $4^{\circ} \mathrm{C}$ using a sandwich approach. After a brief wash with PBS (1×), 
slide tissues were treated with secondary biotinylated-labeled antibodies (Vectastain) for $30 \mathrm{~min}$. The slides were then treated with $\mathrm{ABC}$ reagent (Vectastain Elite ABC kit, Vector Laboratories) for $30 \mathrm{~min}$, after which 3,3-diaminobenzidine (DAB) was added for color development. The slides were examined using white light microscopy (Carl Zeiss Microimaging Inc., Jena, Germany).

\section{Statistical analysis}

Data were presented as mean \pm SD. Comparison between the two groups was performed using a Student's $t$-test, while one-way analysis of variance (ANOVA) (Prism software, GraphPad Software, Inc., La Jolla, CA, USA) was used to compare more than two groups. In both cases, the significance was set at $95 \%$ of confidence.

\section{Results}

\section{Nanobeacon emits strong fluorescent signal suitable for tissue imaging}

The signal intensity of the nanobeacon was quantified using the software available from the in vivo imaging system (IVIS). A dose-dependent emitted photons suggested the probe can be used for quantitative imaging (Figure 2A and B). Based on this information, any concentration starting from $2 \mathrm{mg} / \mathrm{mL}$ is suitable for imaging application. Further, the probe is stable in medium used in clinical settings. Incubation of the nanobeacon in MiraLAX, a laxative prep solution, does not affect the integrity of the nanobeacon (Figure 2C). Notably, the nanobeacon can selectively detect TF expression on frozen human polyp specimens (Figure 2D) $(P=0.0003)$.

\section{The nanobeacon discerns adenoma and adenocarcinoma from normal tissues in a BSA null protocol}

The objective of this experiment was to test whether the nanobeacon could detect the early onset of CRC in unfrozen tissues. Another objective was to determine the potential use of the nanobeacon to replace IHC in the operating room to confirm the early pathological status of dissected tissues. Since this experiment represented the prelude to future application of the nanobeacon in clinical fluorescence colonoscopy, tissue exposure to the nanobeacon remained both simple and practical. The process was tailored specifically to ensure its adaptability to the functions available in the clinical fluorescent colonoscopic imaging system, such as the washing and suction systems used to cleanse and remove the solution. Freshly removed polyps obtained via colectomy were treated with the nanobeacon. To ensure effective detection, particularly the early detection of the disease since cancer may develop from the inner core of an aberrant mass, the tissues were dissected into halves before being exposed briefly to the nanobeacon solution. The data showed that at a concentration of $10 \mathrm{mg} / \mathrm{mL}$ the nanobeacon could detect adenoma or adenocarcinoma in the dissected colonic tissues compared to their respective control normal colons. As shown in Figure 3A and B, it is
A

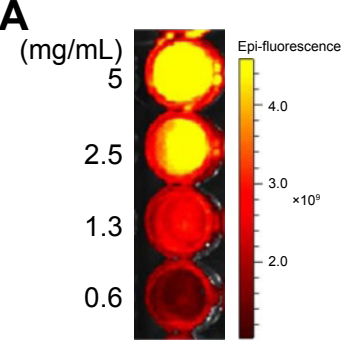

B

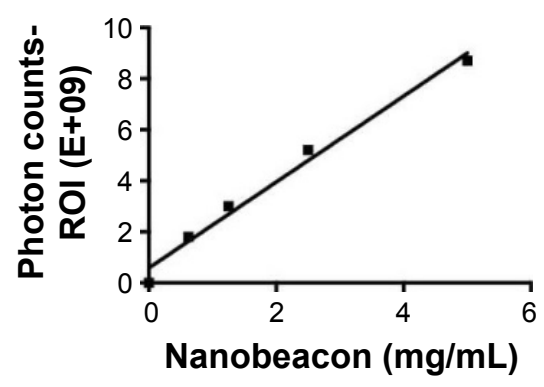

D

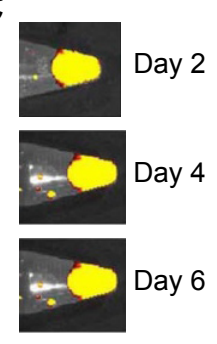

D
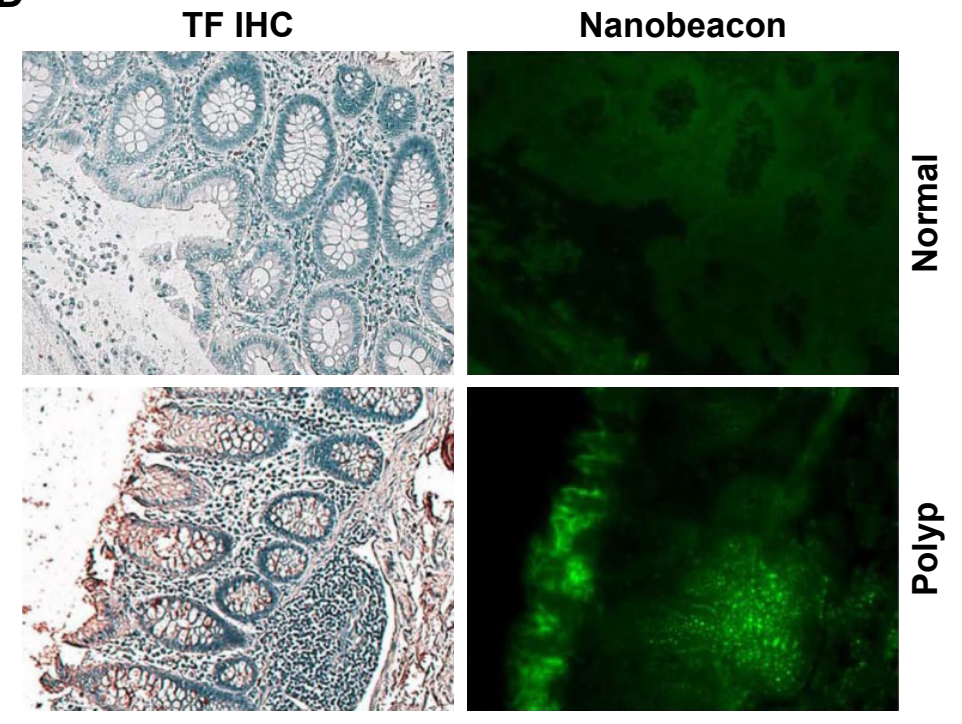

Figure 2 (A) Optical imaging of the nanobeacon using an IVIS to assess the signal strength; (B) linear relationship between concentration and photon counts; (C) signal stability of the nanobeacon was tested in miraLAX solution; (D) the specificity of the nanobeacon to detect TF expression in frozen human colonic specimens ( $P=0.0003$ ). Abbreviations: IVIS, in vivo imaging system; TF, Thomsen-Friedenreich; IHC, immunohistochemical. 

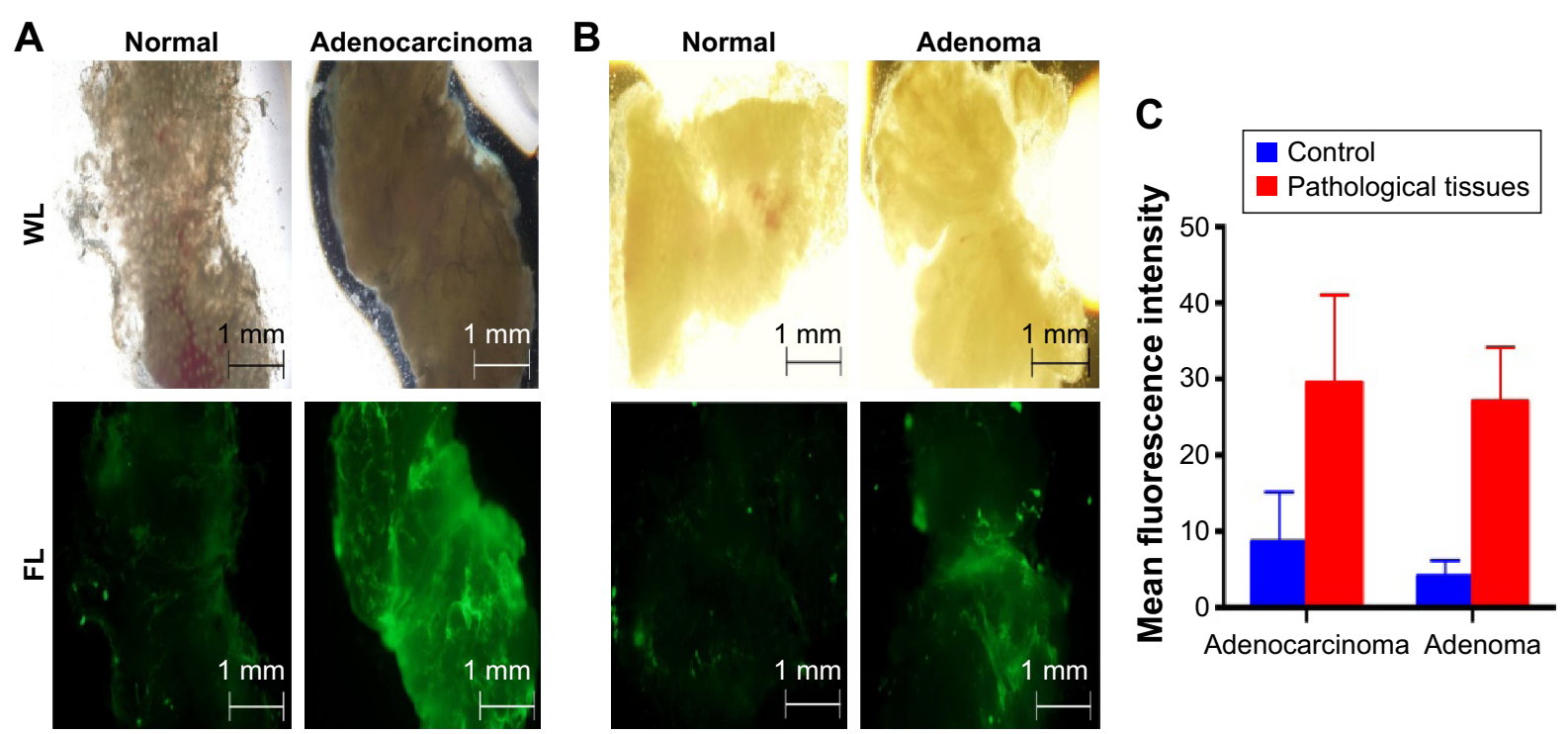

Figure 3 Detection of benign and malignant polyps in a BSA null protocol.

Notes: (A, B) Representative data of freshly dissected colonic tissues from patients ( $\mathrm{n}=8$ pairs) treated with nanobeacon ( $10 \mathrm{mg} / \mathrm{mL})$ briefly before imaging using a fluorescence microscope. Normal and pathological tissues obtained from each patient were dissected and photographed (WL), then washed with PBS ( $4 \times 30$ sec), before being exposed to the nanobeacon ( $1 \mathrm{~min}$ ) before imaging (FL). Magnification $4 \times 10$, exposure time $1 / 60 \mathrm{sec}$. (C) Quantification of the signal intensity emitted from the nanobeacon as a function of tissue characteristics using MATLAB analysis. The data showed significantly increased signals in pathological tissues compared to control $(P<0.05)$.

Abbreviations: BSA, bovine serum albumin; PBS, phosphate buffer saline; WL, white light; FL, fluorescence imaging.

challenging to distinguish normal colonic tissues from their pathological counterparts in the white light mode. However, after brief exposure to the nanobeacon, the fluorescent signal that emanated from pathological tissues was approximately twofold stronger than that emitted by the control counterparts $(P<0.05)$. This observation was made after examining eight pairs (control versus pathological tissues) of biopsied samples (Figure 3C). Of note, most of the tissues we used were no more than $5 \mathrm{~mm}$ in diameter. Further, the work confirms that in this clinical setting, the protocol used to apply the nanobeacon to the tissue is simple and reproducible.

\section{The nanobeacon discerns adenoma and adenocarcinoma from normal tissues in a BSA protocol, albeit with brighter background fluorescence}

Next, we tested the nanobeacon in the protocol that contained a BSA blocking step. In this experiment, 12 pairs of control versus pathological tissues obtained from 12 different individuals were tested. As shown in Figure 4 under the GFP channel, a low and uniform level of background autofluorescence was observed in the background in normal and pathological tissues. However, the binding of the nanobeacon to the highly localized TF antigen expressed on cancerous tissue resulted in a strong and inequitable signal. This led directly to a tangible conclusion about the pathological status of the tissue.
After imaging, the tissues were frozen and prepared for TF-associated IHC staining for corroboration with imaging data. The data in Figure 4 demonstrate that adenoma tissues reported by the nanobeacon were transitioning to undefined, asymmetric, and pencillated cryptic structures arranged in a stratified configuration, which translated to the phenotypically slender stalk shape. Another pathological tissue reported by the nanobeacon was confirmed as adenocarcinoma in the IHC data. Notably, that tissue was very poorly differentiated. Overall, the TF antigen was expressed exclusively and densely in both adenoma and adenocarcinoma tissues; therefore, the imaging data obtained from the nanobeacon corroborated that from the IHC. Altogether, these data suggest that the nanobeacon is capable of detecting the early transforming colonic tissues such as polyps $(P<0.0001)$.

\section{Quantitative analysis of fluorescent histogram in the BSA protocol reveals aberrant tissues}

As the TF antigen is expressed during the early stage of CRC, such as in adenoma cases, the fluorescent signal depicted by the nanobeacon of that expression resulted in clusters of beaconing signals that greatly exceeded those in the background. To exploit that phenomenon, we developed an algorithm to quantify the signal as a secondary method of confirming tissue characteristics. As shown in Figure 5, the fluorescent signals of all control tissues treated with 


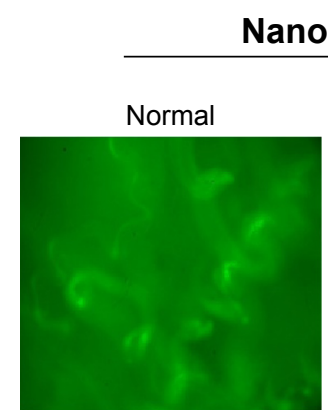

Nanobeacon

Pathological

tissue
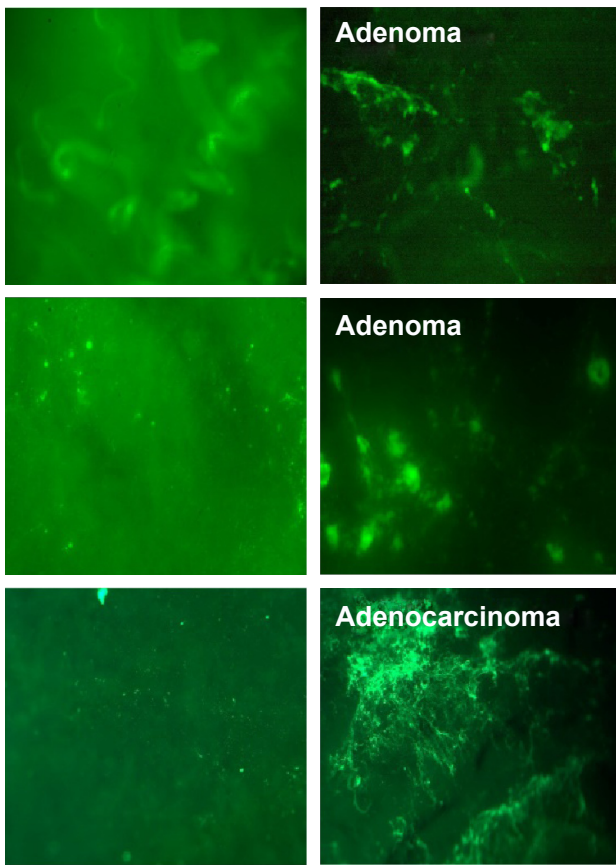
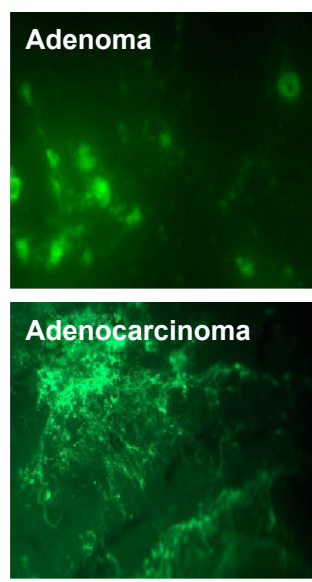

TF histology

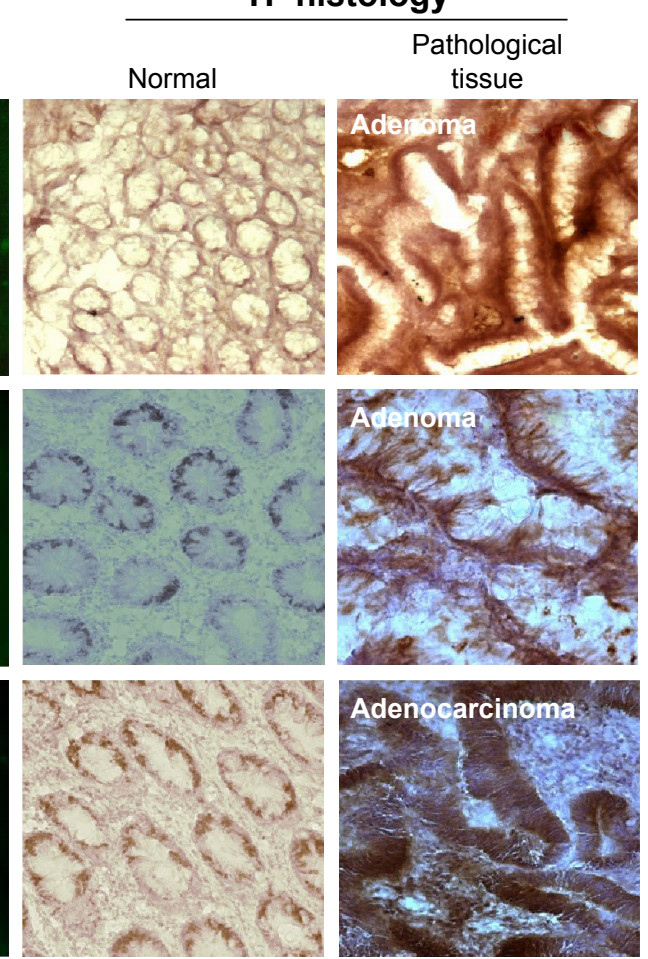

Figure 4 Detection of benign and malignant colonic tissue in a BSA protocol.

Notes: Representative data of freshly biopsied tissues immediately after polypectomy ( $\mathrm{n}=12$ pairs). Normal and pathological tissues were washed with PBS (I min), followed by BSA blocking ( $\mathrm{I} \mathrm{min})$ before being exposed to the nanobeacon ( $\mathrm{I} \mathrm{min})(2 \mathrm{mg} / \mathrm{mL}$, magnification $4 \times$, exposure time $\mid / / 00$ sec). The nonspecific fluorescent signal in the control tissue is significantly reduced $(P<0.000 \mathrm{I})$. Data obtained from the nanobeacon were corroborated with the IHC.

Abbreviations: BSA, bovine serum albumin; PBS, phosphate buffer saline; TF, Thomsen-Friedenreich; IHC, immunohistochemical.

nanobeacon in the BSA protocol were low and uniform throughout the field of view. Thus, their histograms represent an unequivocal Gaussian distribution. In contrast, in aberrant samples the algorithm can successfully delaminate background from TF-associated tissues, resulting in an exponential signal signature.

\section{Discussion}

Our results indicate that the nanobeacon has great potential for the early detection of CRC. As a common malignancy in economically developed countries, particularly in North America, Europe, and Australia, CRC has emerged as one of the leading causes of cancer-related death in the Western world. ${ }^{11}$ Different from other types of cancer, CRC undergoes a protracted asymptomatic stage before it reaches the advanced stage. Thus, it offers a unique window of opportunity for intervention. Successfully detecting the early onset of the disease via routine screening will therefore improve therapeutic outcomes and save lives. ${ }^{9}$ The data presented in this work demonstrated that by integrating a multivalent nanobeacon with quantitative molecular imaging, we can detect and distinguish adenoma and adenocarcinoma from normal colonic tissues in a timely fashion using a simple approach that does not require the use of histology. During the course of this work, we also tested a small number of colonic carcinomas and confirmed the specificity of the nanobeacon (data not shown). Nevertheless, conventional clinical colonoscopy can detect carcinomas without the need for contrasting agents, as carcinomatous lesions are usually accompanied by recognizable bleeding. From a practical perspective, the nanobeacon demonstrated potential for the clinical detection of flat, small, and hidden polyps, the miss rate of which is $24 \%$ in current clinical studies. ${ }^{2}$

Aside from offering special nanoscale physics and multivalency, both of which promote bioconjugation for targeted imaging, the core of each nanobeacon encapsulates thousands of fluorescent dyes, enabling the nanobeacon to emit an especially strong fluorescent signal, which facilitates the imaging of freshly biopsied polyps at reasonably low concentrations. Another advantage of the nanobeacon is that it is intended for topical application. This is especially important since TF antigen is a surface tumor antigen, and fluorescent colonoscopy is an imaging technique that relies on surface surveillance of the colon. Further, most colonoscopy devices in clinical use are 

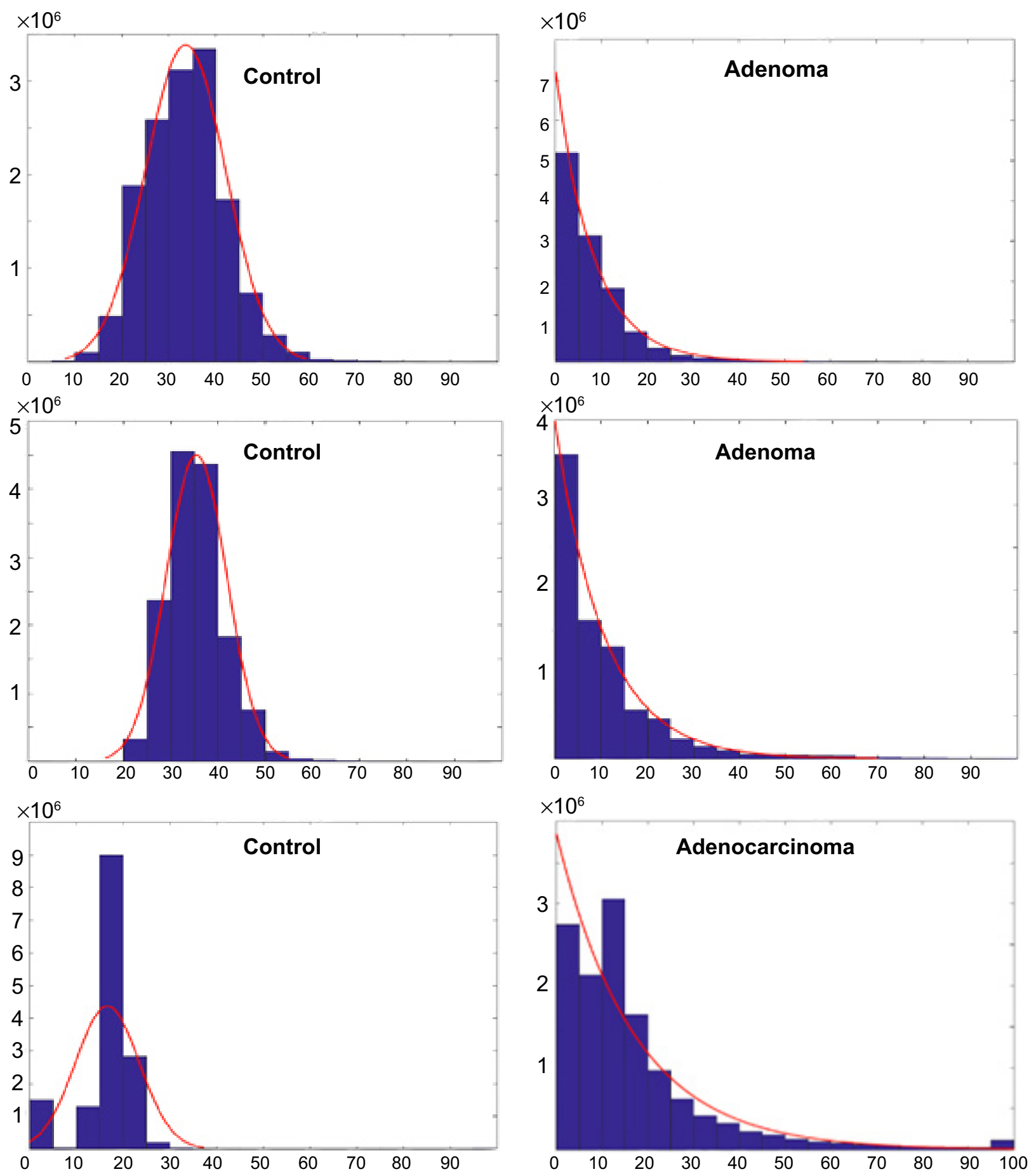

Figure 5 Quantitative analysis of the fluorescent signal in a BSA protocol.

Notes: Representative data of the control, adenoma, and adenocarcinoma tissues ( $n=12$ pairs) after the fluorescent signals were quantified using the in-house developed program. The low and uniform background signal from control tissue fits in a Gaussian distribution curve, while pathological tissues delineate the signal associated with TF association from the background resulting in exponential distribution.

Abbreviations: BSA, bovine serum albumin; TF, Thomsen-Friedenreich.

equipped with spraying/suction devices that are compatible with the process used in nanobeacon application. We reported previously that the hydrodynamic size of the nanobeacon was fine-tuned to prevent its absorption by colonic mucosa. Thus, no systemic distribution occurs in the process, ${ }^{9}$ and toxicity to peripheral organs is avoided. Altogether, we believe the incorporation of a topical nanobeacon with the evolving field of fluorescent colonoscopy technology is a perfect combination.

Despite the potential for clinical translation, reproducibility of the colloidal syntheses is far more challenging 
than small organic syntheses. A few caveats must also be addressed. For instance, we anticipate additional work, such as the implementation of chemical conditions and the establishment of robust quality controls to ensure that different synthesis products will yield nanobeacons that are identical in terms of physical character, size, and number of PNA attached on the surface. Alternatively, additional study is needed to explore whether and to what degree those variables start to affect the signal variability. With the advent of biomedical research that has introduced high-resolution optical imaging techniques ${ }^{12-16}$ now being tested in clinical trials, ${ }^{17}$ the nanobeacon is an indispensable component. For instance, with the recent US Food and Drug Administration (FDA) approval of DNA-based CRC detection in the stool, it is clear that the kit might reveal a positive outcome via DNA detection. However, this alone does not inform patients of the colonic location of the cancer. As a result, patients must still undergo colonoscopy. Since DNA detection is exceptionally sensitive, the malignancy detected is both in the early onset stage and extremely small. As a result, these conditions may challenge the current state of clinical colonoscopy. Thus, integrating fluorescent colonoscopy with molecular biomarker imaging is likely to give an impetus to a shift toward our approach to eradicating this disease.

\section{Acknowledgments}

The authors thank Vanderbilt-based CHTN Western Division for its support, and Osakafu Saiseikai Nakatsu Hospital for providing ex-vivo biopsied tissues and corresponding pathological reports.

\section{Disclosure}

This work was partially funded by grants, R01CA160700 (WP), VICC Cancer Center Support Grant (WP). The authors report no conflicts of interest in this work.

\section{References}

1. Daley D, Lewis S, Platzer P, et al. Identification of susceptibility genes for cancer in a genome-wide scan: results from the colon neoplasia sibling study. Am J Hum Genet. 2008;82:723-736.

2. Rex DK, Cutler CS, Lemmel GT, et al. Colonoscopic miss rates of adenomas determined by back-to-back colonoscopies. Gastroenterology. 1997;112:24-28.
3. Villavicencio RT, Rex DK. Colonic adenomas: prevalence and incidence rates, growth rates, and miss rates at colonoscopy. Semin Gastrointest Dis. 2000;11:185-193.

4. Bujanda L, Cosme A, Gil I, Arenas-Mirave JI. Malignant colorectal polyps. World J Gastroenterol. 2010;16:3103-3111.

5. Eaden JA, Mayberry JF. Guidelines for screening and surveillance of asymptomatic colorectal cancer in patients with inflammatory bowel disease. Gut. 2002;51(Suppl 5):V10-V12.

6. Brockhausen I. Mucin-type O-glycans in human colon and breast cancer: glycodynamics and functions. EMBO Rep. 2006;7:599-604.

7. Baldus SE, Hanisch FG, Kotlarek GM, et al. Coexpression of MUC1 mucin peptide core and the Thomsen-Friedenreich antigen in colorectal neoplasms. Cancer. 1998;82:1019-1027.

8. Cao Y, Karsten UR, Liebrich W, Haensch W, Springer GF, Schlag PM. Expression of Thomsen-Friedenreich-related antigens in primary and metastatic colorectal carcinomas. A reevaluation. Cancer. 1995;76: $1700-1708$

9. Kumagai H, Pham W, Kataoka M, et al. Multifunctional nanobeacon for imaging Thomsen-Friedenreich antigen-associated colorectal cancer. Int J Cancer. 2013;132:2107-2117.

10. Sakuma S, Yu JY, Quang T, et al. Fluorescence-based endoscopic imaging of Thomsen-Friedenreich antigen to improve early detection of colorectal cancer. Int J Cancer. 2015;136:1095-1103.

11. Subramaniam D, May R, Sureban SM, et al. Diphenyl difluoroketone: a curcumin derivative with potent in vivo anticancer activity. Cancer Res. 2008;68:1962-1969.

12. Iftimia N, Cizginer S, Deshpande V, et al. Differentiation of pancreatic cysts with optical coherence tomography (OCT) imaging: an ex vivo pilot study. Biomed Opt Express. 2011;2:2372-2382.

13. Maguluri G, Mujat M, Park BH, et al. Three dimensional tracking for volumetric spectral-domain optical coherence tomography. Opt Express. 2007; 15:16808-16817.

14. Mastanduno MA, El-Ghussein F, Jiang S, et al. Adaptable near-infrared spectroscopy fiber array for improved coupling to different breast sizes during clinical MRI. Acad Radiol. 2014;21:141-150.

15. Tromberg BJ, Cerussi A, Shah N, et al. Imaging in breast cancer: diffuse optics in breast cancer: detecting tumors in pre-menopausal women and monitoring neoadjuvant chemotherapy. Breast Cancer Res. 2005;7: 279-285.

16. Valdes PA, Jacobs VL, Wilson BC, Leblond F, Roberts DW, Paulsen KD. System and methods for wide-field quantitative fluorescence imaging during neurosurgery. Opt Lett. 2013;38:2786-2788.

17. Wang J, Jiang S, Li Z, et al. In vivo quantitative imaging of normal and cancerous breast tissue using broadband diffuse optical tomography. Med Phys. 2010;37:3715-3724. 


\section{Supplementary material}

\begin{tabular}{|c|c|c|c|c|c|}
\hline \multicolumn{6}{|c|}{ Supporting information } \\
\hline \multirow[t]{2}{*}{ Inspection item } & \multirow[t]{2}{*}{ Unit } & \multirow{2}{*}{$\frac{2014 \text { lot }}{\text { MDO1914A }}$} & \multicolumn{2}{|l|}{2015 lot } & \multirow{2}{*}{$\frac{2016 \text { lot }}{\text { NBS-071 }}$} \\
\hline & & & MO23315A & M018315A & \\
\hline MW (PNVAM) & MW & 16,900 & 16,900 & 16,900 & 21,700 \\
\hline MW (PMAM) & MW & 11,900 & 11,900 & 11,900 & 10,500 \\
\hline Particle size & $\mathrm{nm}$ & 360 & 340 & 350 & 450 \\
\hline Fluorescent intensity & $\mathrm{AU}$ & $2.6 \times 10^{5}$ & $3.1 \times 10^{5}$ & $3.2 \times 10^{5}$ & $3.5 \times 10^{5}$ \\
\hline PNA content & $\mu \mathrm{g} / \mathrm{mg} \mathrm{NB}$ & 3.0 & 3.5 & 3.6 & 6.4 \\
\hline
\end{tabular}

Figure SI Different batches of PNA-laden nanobeacon were characterized to confirm the reproducibility of the chemistry.

Abbreviations: PNA, peanut agglutinin; PNVAM, poly(N-vinylacetamide) macromonomer; PMAM, poly(methacrylic acid) macromonomer.

\section{Publish your work in this journal}

The International Journal of Nanomedicine is an international, peerreviewed journal focusing on the application of nanotechnology in diagnostics, therapeutics, and drug delivery systems throughout the biomedical field. This journal is indexed on PubMed Central, MedLine, CAS, SciSearch ${ }^{\circledR}$, Current Contents ${ }^{\circledR} /$ Clinical Medicine,
Journal Citation Reports/Science Edition, EMBase, Scopus and the Elsevier Bibliographic databases. The manuscript management system is completely online and includes a very quick and fair peer-review system, which is all easy to use. Visit http://www.dovepress.com/ testimonials.php to read real quotes from published authors.

\footnotetext{
Submit your manuscript here: http://www.dovepress.com/international-journal-of-nanomedicine-journal
} 